\title{
Electromagnetic enhancement of turbulent heat transfer
}

\author{
Saša Kenjerě̌ \\ Department of Multi-Scale Physics and J.M. Burgers Centre for Fluid Dynamics, Delft University of Technology, \\ Prins Bernhardlaan 6, 2628 BW Delft, The Netherlands
}

(Received 23 July 2008; published 18 December 2008)

\begin{abstract}
We performed large eddy simulations (LES) of the turbulent natural convection of an electrically conductive fluid (water with $7 \% \mathrm{Na}_{2} \mathrm{SO}_{4}$ electrolyte solution) in a moderate (4:4:1) aspect ratio enclosure heated from below and cooled from above and subjected to external nonuniformly distributed electromagnetic fields. Different configurations with permanent magnets (located under the lower thermally active wall, $B_{0}=1 \mathrm{~T}$ ) and different strengths of imposed dc electric currents $(I=0-10 \mathrm{~A})$ were compared to the case of pure thermal convection in the turbulent regime, $\operatorname{Ra}=10^{7}, \operatorname{Pr}=7$. It is demonstrated that the electromagnetic forcing of the boundary layers caused significant reorganization of flow and turbulence structures producing significant enhancement of the wall-heat transfer (up to $188 \%$ for a configuration with 35 magnets and an applied dc current of $10 \mathrm{~A}$ ).
\end{abstract}

DOI: 10.1103/PhysRevE.78.066309

PACS number(s): 47.27.- i, 47.65.- d, 47.85.- $-\mathrm{g}$

\section{INTRODUCTION}

Control of flow, turbulence, and heat transfer of electrically conductive working fluids is the basic prerequisite for the design and optimization of many technological processes. Examples include electromagnetic braking of continuous steel casting, free-surface stabilization of the aluminum reduction cells, joining metals by arc welding, crystal growth, electromagnetic mixing, and steering in metallurgy and liquid metal blankets in new generation of fusion reactors $[1-3]$.

In previous studies, we analyzed the effects of imposed uniform magnetic fields of different orientation and strength on turbulent forced and thermal convection of highly electrically conductive fluids [4-7]. It was observed that the resulting Lorentz force significantly affected the underlying flow, turbulence, and wall-heat transfer. The wall-heat transfer was strongly reduced for a vertically oriented magnetic field, making it possible to totally suppress convective motions for sufficiently strong magnetic fields. The numerical simulations proved to provide results in very good agreement with available experiments over a range of Rayleigh $(\mathrm{Ra})$, Prandtl (Pr), and Hartmann (Ha) numbers [8], where $\mathrm{Ra}$ $=\beta g \Delta T \operatorname{Pr} \rho^{2} H^{3} / \mu^{2}, \operatorname{Pr}=\nu / a$, and $\mathrm{Ha}=\left|B_{0}\right| H \sqrt{\sigma / \rho \nu}$. For a longitudinal orientation of the magnetic field, a twodimensional alignment of flow structures with the direction of the imposed magnetic field is observed. When a full twodimensionality of the flow is reached (total suppression of the flow variations in the spanwise direction), a saturation point in the effective reduction of the wall-heat transfer is achieved [6].

\section{INVESTIGATED SETUP}

In the present investigation, instead of applying uniformly distributed magnetic fields, we focus on the possibility to locally apply electromagnetic forcing in order to affect the boundary layers along the thermally active horizontal walls. In order to achieve that, different combinations of magnets and electrodes are imposed, resulting in various forcing patterns on the underlying flow. In contrast to the multiscale forcing applied in Refs. [9,10], where laminar twodimensional flow patterns have been investigated, we focus on genuinely three-dimensional flow features in fully developed turbulent regimes with heat transfer. The heat transfer was not considered in our previous experimental and numerical studies of electromagnetically driven flows [11]. The noslip boundary condition for velocity is applied for all enclosure walls and horizontal walls (upper cold, lower hot) are kept at the constant temperature. The all side walls are adiabatic. Special care is taken to choose simulation parameters close to experimentally reachable flow regimes. The turbulent thermal convection at $\mathrm{Ra}=10^{7}, \mathrm{Pr}=7$ is selected as a referent case. Despite the relatively weak electrical conductivity of the working fluid that is close to that of sea water (water with $7 \% \mathrm{Na}_{2} \mathrm{SO}_{4}, \sigma=5.551 / \Omega \mathrm{m}$ ), sufficiently strong electromagnetic forces can be generated in the proximity of the lower wall with support of the imposed electric dc current (distributed through two electrodes located at the top of the side walls). The two-magnet configuration generates a well-defined central vortical tornadolike flow pattern similar to those presented in Ref. [12]. The three-magnet configuration, the central alignment of which is rotated over $90^{\circ}$ with respect to the two-magnet configuration, generates local flow patterns in the form of a horizontal walljet along the lower wall [11]. Finally, an array of 35 magnets generates complex flow patterns that result from superposition of the above mentioned magnetic orientations.

\section{NUMERICAL MODEL}

The flow of an incompressible electrically conductive working fluid in the turbulent regime, subjected to temperature gradients and Lorentz force, can be described by conservation of momentum and heat: 


$$
\begin{aligned}
& \frac{\partial\left\langle U_{i}\right\rangle}{\partial t}+\left\langle U_{j}\right\rangle \frac{\partial\left\langle U_{i}\right\rangle}{\partial x_{j}}= \frac{\partial}{\partial x_{j}}\left(\nu \frac{\partial\left\langle U_{i}\right\rangle}{\partial x_{j}}-\tau_{i j}\right) \\
&-\frac{1}{\rho} \frac{\partial\langle P\rangle}{\partial x_{i}}-\underbrace{\beta g_{i}\left(\langle T\rangle-T_{\mathrm{REF}}\right)}_{F_{B}}+\underbrace{\frac{\sigma}{\rho} \varepsilon_{i j k}\left\langle E_{j}\right\rangle\left\langle B_{k}\right\rangle}_{F_{L}} \\
& \frac{\partial\langle T\rangle}{\partial t}+\left\langle U_{j}\right\rangle \frac{\partial\langle T\rangle}{\partial x_{j}}=\frac{\partial}{\partial x_{j}}\left(\frac{\nu}{\operatorname{Pr}} \frac{\partial\langle T\rangle}{\partial x_{j}}-\tau_{\theta j}\right)
\end{aligned}
$$

together with the solenoidal conditions $\partial\left\langle U_{i}\right\rangle / \partial x_{i}=0$ and $\partial\left\langle B_{i}\right\rangle / \partial x_{i}=0$. Here, " $\langle\cdots\rangle$ " stands for the spatially averaged (filtered) quantities in a large eddy simulation approach. In these equations, $\left\langle U_{i}, P, T, E_{i}, B_{i}\right\rangle$ are velocity, pressure, temperature, electric field, and magnetic field, respectively. The magnetic and electric field distributions are calculated from the simplified set of Maxwell's equations by applying the Biot-Savart law for permanent magnets and electrodes. For the considered flow regimes, the inductive current contributions $(\mathbf{U} \times \mathbf{B})$ to the total electric current density $\mathbf{J}=\sigma(\mathbf{E}$ $+\mathbf{U} \times \mathbf{B})$ are negligible compared to the conductive current, resulting in a Lorentz force $\left(F_{L}\right)$ that is constant in time. The subgrid turbulence contributions $\tau_{i j}$ and $\tau_{\theta i}$ are calculated from

$$
\begin{gathered}
\tau_{i j}=\frac{1}{3} \tau_{k k} \delta_{i j}-\nu_{t}\left\langle S_{i j}\right\rangle, \quad \tau_{\theta i}=\frac{\nu_{t}}{\operatorname{Pr}_{t}} \frac{\partial\langle T\rangle}{\partial x_{i}}, \\
\nu_{t}=\nu_{s} \exp \left[-\left(\frac{\sigma}{\rho}\right)\left(C_{m} \Delta\right)^{2}\left|B_{0}\right|^{2} / \nu_{s}\right], \\
\nu_{s}=\left(C_{s} \Delta\right)^{2}\left(\left\langle S_{i j}\right\rangle\left\langle S_{i j}\right\rangle\right)^{1 / 2}, \\
\left\langle S_{i j}\right\rangle=\frac{1}{2}\left(\frac{\partial\left\langle U_{i}\right\rangle}{\partial x_{j}}+\frac{\partial\left\langle U_{j}\right\rangle}{\partial x_{i}}\right), \\
C_{s}=C_{s 0}\left[1-\exp \left(\frac{-z_{n}^{+}}{25}\right)\right] .
\end{gathered}
$$

Here, the turbulent subgrid viscosity $\left(\nu_{t}\right)$ is evaluated from the magnetically extended Smagorinsky model in order to account for additional magnetic suppression of velocity fluctuations, as proposed in Ref. [13]. The subgrid turbulence model constants are $C_{s 0}=0.1$ and $C_{m}=1.4$. The subgrid turbulent heat flux $\left(\tau_{\theta i}\right)$ is calculated from the simple-gradient diffusion hypothesis with the turbulent Prandtl number $\operatorname{Pr}_{t}$ $=0.9$. In order to provide proper near-wall behavior of the turbulent viscosity, the van Driest damping function is used in the proximity of the walls (for both side and thermally active walls, where $z_{n}^{+}$is the nondimensional wall distance). The set of equations Eqs. (1)-(5) is discretized by using a second-order implicit finite-volume Navier-Stokes +Maxwell solver for three-dimensional nonorthogonal geometries. Typical values of the time steps are used to ensure that the maximum Courant-Friedrichs-Levy number will be 0.5 . About $7.5 \times 10^{4}$ instantaneous fields (representing 15-70 characteristic eddy turnover times, Kerr [17], for neutral and electromagnetic forcing cases, respectively) are used to obtain convergent statistics of the second moments. All additional details of numerical solver can be found in Refs. [14-16].

\section{RESULTS}

Prior simulations with heat transfer, a detailed comparison was performed between LES and experimental data [obtained from the particle image velocimetry (PIV) measurements] [11]. A numerical mesh consisting of $182 \times 182$ $\times 92$ control volumes clustered $\left(z_{n}^{+}=0.5\right)$ in the proximity of thermally active and side walls is used. This mesh is designed such that it fully resolves estimated flow and turbulence scales in the proximity of the walls $(1-2) \eta_{K}$ where $\eta_{K}=\left(\nu^{3} / \varepsilon\right)^{1 / 4}$ is the Kolmogorov length scale. A coarser resolution of $(5-10) \eta_{K}$ is used in the central part of the enclosure. These estimates are based on the case for the highest intensity of applied electric current ( $I=10 \mathrm{~A})$. The resulting maximum ratio between subgrid turbulent and molecular viscosity was $\nu_{t} / \nu<0.1$ for the strongest forcing case with 35 magnets, proving sufficiency of the spatial resolution. For the isothermal case, LES results showed good agreement with available PIV data-for velocity and turbulent stresses in two- and three-magnet configurations and different strengths of imposed current [11]. Simulations of pure turbulent thermal convection over a range of $\mathrm{Ra}$ and Pr have been validated in Refs. $[6,14,19,20]$. Now, for the nonisothermal case with electromagnetic fields, distributions of the Lorentz force lines and imposed magnetic field beneath the lower wall $\left(B_{z}\right)$ and resulting flow and temperature patterns (for fully developed flow) are shown in Fig. 1. It can be seen that the generated Lorentz force is mainly concentrated in the lower part of the setup (first $10 \%$ of the total height, Fig. 2), where characteristic spiraling flow patterns are generated. Those locally imposed vortical patterns change the initial morphology of both the thermal and hydrodynamical boundary layers along the horizontal thermally active walls. The difference in the vertical extension of the thermal boundary layers along horizontal walls is clearly visible in vertical planes showing contours of as shown in Fig. 1. Superimposed streamlines and temperature contours of the long-time averaged fields in the central vertical plane for different magnet setups are shown in Fig. 3. Figure 3(a) shows the neutral (no magnet) situation, where two characteristic convective rolls occupy the central part of the cavity and two secondary rolls are located in the upper corners. The convective rolls show very similar morphology to those analyzed in Ref. [20], whereas the temperature field portrays "mushroomlike" thermal plumes that closely resemble structures observed in Refs. $[17,18]$. Although direct forcing by the Lorentz force is locally confined to the boundary layers, due to mutual coupling of velocity and temperature fields (thermal buoyancy), any changes in the near-wall regions lead to relocation of the thermal "mushroomlike" plumes (vertical up drafts of the hot fluid) that in turn define the intensity and size of convective and roll structures. Already for the two-magnet situation, Fig. $3(\mathrm{~b})$, the horizontal extension of the downward thermal plume, originating from the top-cold surface, is significantly 

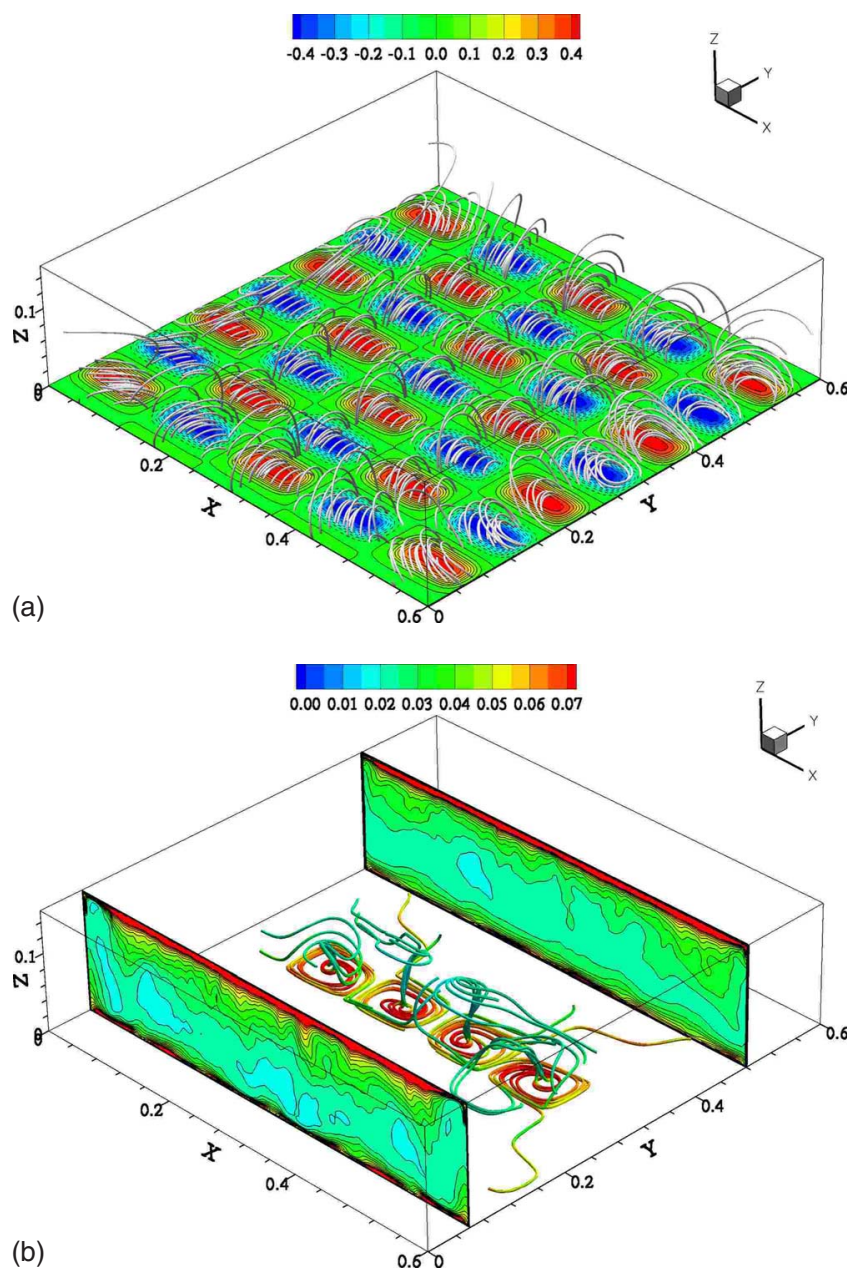

FIG. 1. (Color online) Considered [4:4:1] wall-bounded enclosure $\mathrm{Ra}=10^{7}, \mathrm{Pr}=7$. Above: Contours of the vertical magnetic field component $\left(B_{z}\right.$ in $\left.\mathrm{T}\right)$ at the bottom wall and the Lorentz force line distributions (colored by temperature). Below: tracers of the time averaged velocity field colored by nondimensional temperature variance $\left(\sqrt{\left\langle\theta^{2}\right\rangle} / \Delta T\right)$ and its contours in two characteristic vertical planes. All enclosure dimensions are in $\mathrm{m}$.

reduced due to the centrally located swirling pattern. This swirling pattern creates a low-pressure region that attracts the surrounding fluid and moves convective rolls towards the
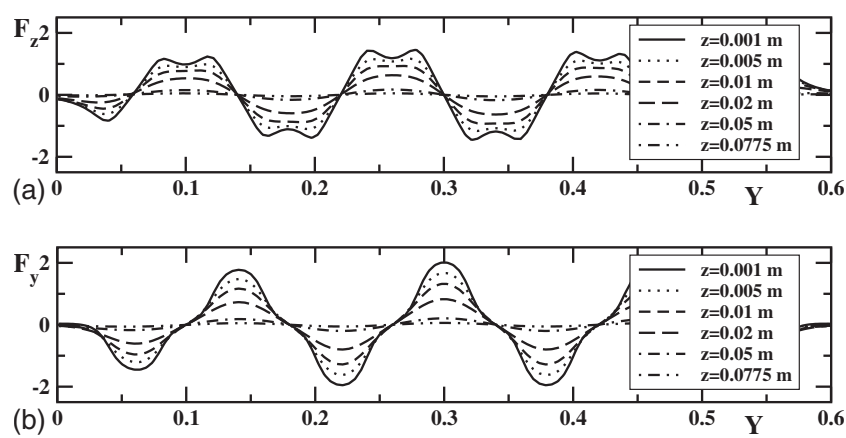

FIG. 2. Profiles of the $z$ and $y$ components of the imposed Lorentz force (in $\mathrm{N} / \mathrm{m}^{3}$ ) in the central vertical plane at different distances from the lower wall for a configuration with 35 magnets $\left(\left|B_{0}\right|=1 \mathrm{~T}\right.$ and $\left.I=1 \mathrm{~A}\right)$.
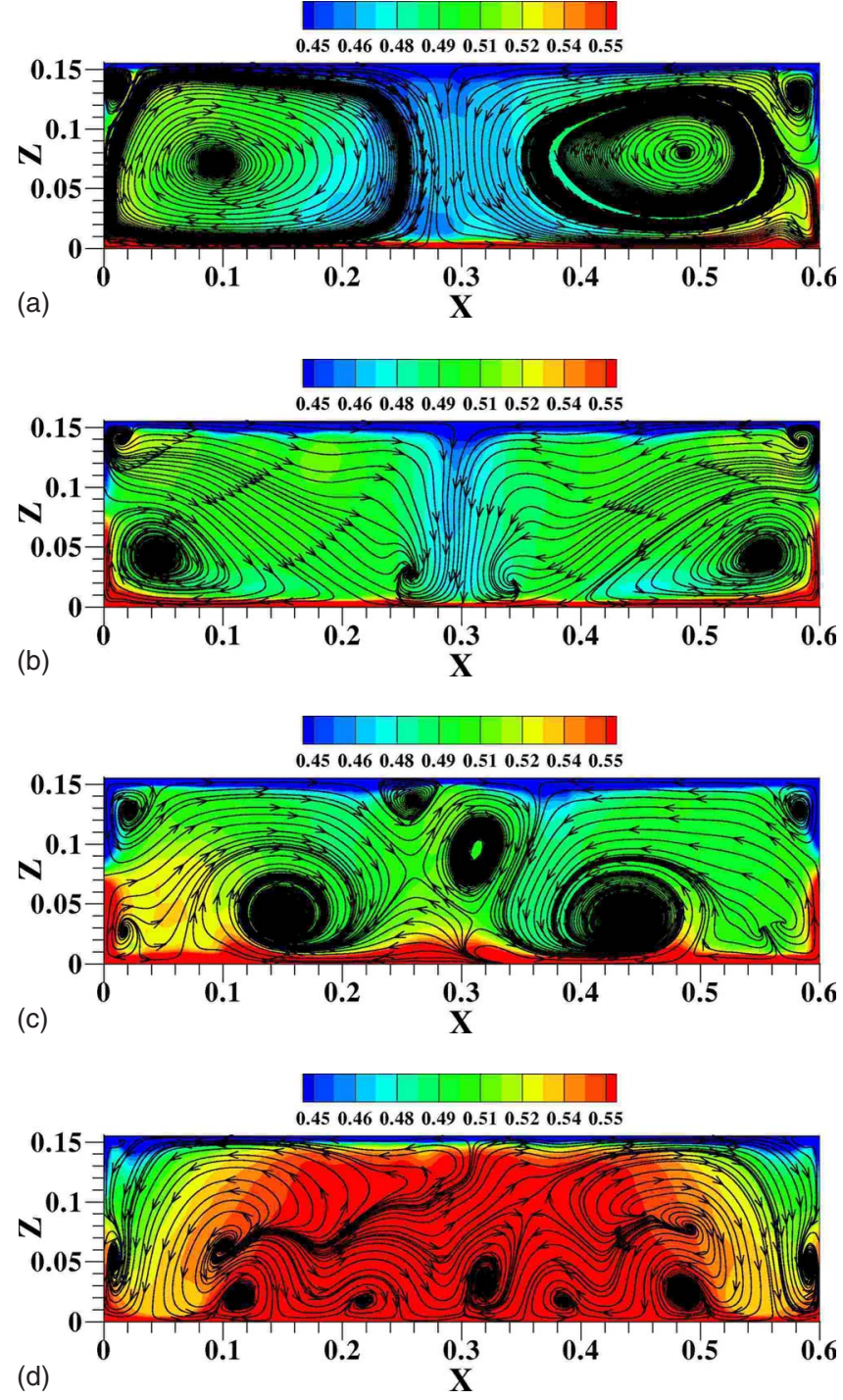

FIG. 3. (Color online) Long-time averaged stream traces and temperature contours in the central vertical plane for configurations with $0,2,3$, and 35 magnets from top to bottom, respectively. I $=1 \mathrm{~A}, \mathrm{Ra}=10^{7}, \operatorname{Pr}=7$.

lower side corners, reducing their size. In contrast to this "central forcing," the configurations with 3 and 35 magnets produce multiple cells in the proximity of the lower horizontal wall, Figs. 3(c) and 3(d). Vertical long-time and spatially averaged temperature profiles show an interesting shift toward higher values in the central part of the enclosure when electromagnetic control is active, Fig. 4(a) and 4(b). This is a consequence of the asymmetrical forcing since only the lower part of the enclosure is subjected to the locally distributed Lorentz force. Compared to the neutral situation, the temperature variance profiles $(\langle\bar{\theta}\rangle)$ exhibit lower peaks and are moved closer to the lower wall, indicating thinning of the thermal boundary layer, Fig. 4(c) and 4(d). In contrast to that, in the proximity of the upper wall, the temperature variance profiles show higher peak values compared to the neutral situation. The spatially averaged horizontal velocity component $(\langle V\rangle)$ exhibits changes in the sign and in the magnitude for the 2- and 35-magnet configuration and strong (I 

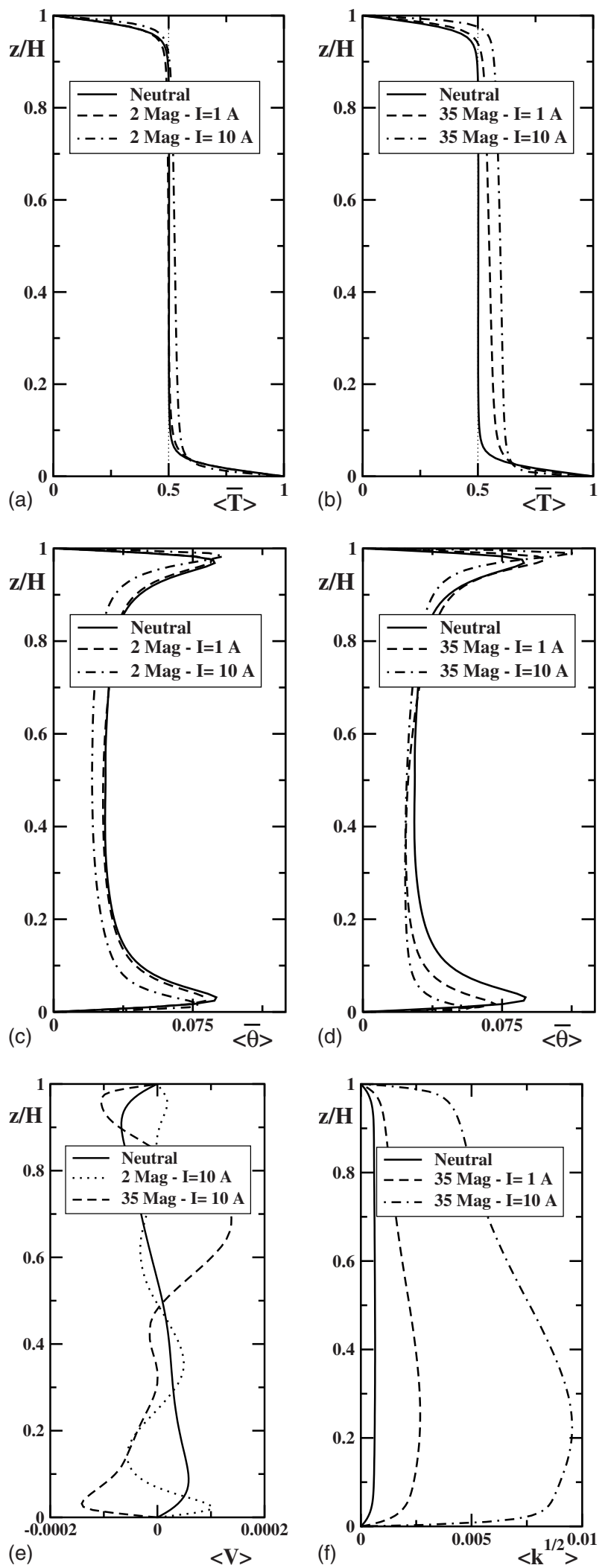

FIG. 4. (Color online) Long-time and spatially averaged vertical profiles of temperature $(\mathrm{a}-\mathrm{b})$, temperature variance $(\mathrm{c}-\mathrm{d})$, horizontal velocity $\langle V\rangle$ (nondimensionalized by $U_{b}=\sqrt{\beta g \Delta T H}$ ) (e), and kinetic energy of turbulence (f) for 2 and 35 magnet configurations and different intensities of applied electric currents $I=0,1,10 \mathrm{~A}$, respectively.
TABLE I. Nusselt numbers for different magnet configurations and different strengths of applied electric currents. The lower and upper range of $\mathrm{Re}$ are for weak (1 A) and strong (10 A) currents, respectively. $\mathrm{Ra}=10^{7}, \mathrm{Pr}=7, \mathrm{Ha}=10,\left|B_{0}\right|=1 \mathrm{~T}$.

\begin{tabular}{lccc}
\hline \hline Configuration & Applied dc: $I=1 \mathrm{~A}$ & $=10 \mathrm{~A}$ & $\mathrm{Re}^{\mathrm{a}}$ \\
\hline Neutral & 15.35 & 15.35 & 200 \\
2 magnets & $16.27(+6 \%)$ & $25.75(+68 \%)$ & $1200-4000$ \\
3 magnets & $15.70(+2.3 \%)$ & $25.05(+63 \%)$ & $900-3000$ \\
35 magnets & $20.58(+34 \%)$ & $44.28(+188 \%)$ & $1300-4500$ \\
\hline \hline
\end{tabular}

${ }^{\mathrm{a}} \operatorname{Re}=\max \left|\left\langle U_{i}\right\rangle\right| H / \nu,\left|U_{i}\right|=\sqrt{\langle U\rangle^{2}+\langle V\rangle^{2}+\langle W\rangle^{2}}$.

$=10$ A) current, Fig. 4(e). The turbulence kinetic energy profiles show a big increase over the entire enclosure height, with a characteristic peak in the proximity of the lower wall, Fig. 4(f). Again, strong asymmetry is clearly visibleespecially for higher intensities of applied dc current.

Finally, a summary of Nusselt numbers for various magnet configurations and intensities of electric currents is given in Table I. The value of $\mathrm{Nu}=15.35$ for the neutral situation agrees well with experimentally obtained correlations $\mathrm{Nu}$ $=0.145 \mathrm{Ra}^{0.29}=15.54[21]$ and $\mathrm{Nu}=0.183 \mathrm{Ra}^{0.278}=16.1[22]$. Also good agreement is obtained with DNS results of $\mathrm{Nu}$ $=15.75$ [23]. It can be seen that flow and turbulence reorganization in the presence of external electromagnetic fields has a significant impact on the wall-heat transfer. For all considered combinations, an enhancement in $\mathrm{Nu}$ is observed-ranging from slightly increased values for twoand three-magnet configurations and weak electric currents to very large values for stronger electric currents. It is interesting to note that the two-magnet configuration is more efficient than the three-magnet configuration, since it imposes direct shear forcing in the lower part of the enclosure that finally creates a local low-pressure region with a swirling flow pattern that vertically elongates the central thermal mushroomlike plume. The significance of the obtained heat transfer enhancement can be compared with values obtained in Ref. [24], where an enhancement of 5-10\% was experimentally obtained in annular turbulent flow $\left(5 \times 10^{3} \leqslant \mathrm{Re}\right.$ $\left.\leqslant 10^{5}\right)$ of lithium subjected to a magnetic field $\left(\left|B_{0}\right|\right.$ $\leqslant 0.8 \mathrm{~T})$.

\section{CONCLUSION}

It was demonstrated that application of a steady, locally distributed Lorentz force can produce a very large increase in the wall-heat transfer in turbulent thermal convection. In addition to its potential in various technological applications, this localized electromagnetic forcing can be used for fundamental studies of turbulence subjected to external body forces (modulated turbulence).

\section{ACKNOWLEDGMENTS}

The high-performance computing facilities were provided by the NWO/NCF and SARA Computational and Network services, Amsterdam, The Netherlands [25]. 
[1] R. Moreau, Magnetohydrodynamics (Kluwer Academic, Dordrecht, 1990).

[2] P. A. Davidson, Annu. Rev. Fluid Mech. 31, 273 (1999).

[3] P. A. Davidson, An Introduction to Magnetohydrodynamics (Cambridge University Press, Cambridge, 2001).

[4] K. Hanjalić and S. Kenjereš, J. Turbul. 1, 1 (2000).

[5] K. Hanjalić and S. Kenjereš, Flow, Turbul. Combust. 66, 427 (2001).

[6] S. Kenjereš and K. Hanjalić, Int. J. Heat Fluid Flow 25, 559 (2004).

[7] S. Kenjereš, K. Hanjalić, and D. Bal, Phys. Fluids 16, 1229 (2004).

[8] S. Cioni, S. Chaumat, and J. Sommeria, Phys. Rev. E 62, R4520 (2000).

[9] L. Rossi, J. C. Vassilicos, and Y. Hardalupas, Phys. Rev. Lett. 97, 144501 (2006).

[10] L. Rossi, J. C. Vassilicos, and Y. Hardalupas, J. Fluid Mech. 558, 207 (2006).

[11] S. Kenjereš, J. Verdoold, A. Wibowo, C. R. Kleijn, and K. Hanjalić, Advances in Turbulence XI, edited by J. M. L. M. Palma and A. S. Lopes, Vol. 117 of Springer Proceedings in
Physics (Springer, Berlin, 2007), pp. 656-658.

[12] C. S. Yih, Phys. Fluids 19, 076601 (2007).

[13] Y. Shimomura, Phys. Fluids A 3, 3098 (1991).

[14] S. Kenjereš, K. Hanjalić, S. Renaudier, F. Stefani, G. Gerbeth, and A. Gailitis, Phys. Plasmas 13, 122308 (2006).

[15] S. Kenjereš and K. Hanjalić, Phys. Rev. Lett. 98, 104501 (2007).

[16] S. Kenjereš and K. Hanjalić, New J. Phys. 9, 1 (2007).

[17] R. M. Kerr, J. Fluid Mech. 310, 139 (1996).

[18] Q. Zhou, C. Sun, and K.-Q. Xia, Phys. Rev. Lett. 98, 074501 (2007).

[19] S. Kenjereš and K. Hanjalić, Phys. Rev. E 66, 036307 (2002).

[20] S. Kenjereš and K. Hanjalić, Phys. Rev. E 62, 7987 (2000).

[21] H. Tanaka and H. Miyata, Int. J. Heat Mass Transfer 23, 1273 (1980).

[22] T. Chu and R. J. Goldstein, J. Fluid Mech. 60, 141 (1973).

[23] R. M. Kerr and J. R. Herring, J. Fluid Mech. 419, 325 (2000).

[24] N. Uda, N. Yamaoke, H. Horiike, and K. Miyazaki, Energy Convers. Manage. 43, 441 (2002).

[25] http://www.sara.nl 